\title{
Searching for Pulsars in the WENSS
}

\author{
Marco Kouwenhoven \\ Astronomical Institute, Utrecht University \\ Michiel Berger \\ Astronomical Institute, University of Amsterdam \\ Will Deich, Ger de Bruyn \\ Netherlands Foundation for Research in Astronomy
}

\section{What is the WENSS?}

The Westerbork Northern Sky Survey is an imaging survey carried out at 325 $\mathrm{MHz}$ by the Westerbork Synthesis Array Telescope (de Bruyn, 1996). It covers the northern sky above declination $30^{\circ}$ with a resolution of 54 " and a limiting flux density $(5 \sigma)$ of circa $15 \mathrm{mJy} /$ beam. The WENSS also provides polarization maps of this part of the sky. About one fourth of the WENSS-325 area will also be covered at $608 \mathrm{MHz}$ to the same limiting flux density. Table 1 gives the parameters of these surveys. It also contains the parameters of the FIRST (Faint Images of the Radio Sky at 'Twenty-cm, Becker et al. 1995), which is a high resolution survey carried out at $1500 \mathrm{MHz}$.

Table 1. Parameters of surveys used for selection pulsar candidates.

\begin{tabular}{|c|c|c|c|}
\hline $\begin{array}{r}\text { Survey } \\
\text { Telescope }\end{array}$ & $\begin{array}{c}\text { WENSS-325 } \\
\text { WSRT }\end{array}$ & $\begin{array}{c}\text { WENSS-608 } \\
\text { WSRT }\end{array}$ & $\underset{\text { VLA }}{\text { FIRST }}$ \\
\hline $\begin{array}{r}\text { Frequency } \\
\text { Area } \\
\\
\text { Resolution } \\
\text { Position Accuracy } \\
\text { Noise }\end{array}$ & $\begin{array}{c}325 \mathrm{MHz} \\
\text { Dec } \geq 30^{\circ} \\
54^{\prime \prime} \\
5 " \\
3 \mathrm{mJy}\end{array}$ & $\begin{array}{c}608 \mathrm{MHz} \\
30 \% \text { of WENSS-325 } \\
30 " \\
5^{\prime \prime} \\
3 \mathrm{mJy}\end{array}$ & $\begin{array}{c}1500 \mathrm{MHz} \\
10.000 \text { deg. }^{2} \text { around } \\
\text { North. Gal. Cap } \\
5 " \\
<1 " \\
0.15 \mathrm{mJy}\end{array}$ \\
\hline
\end{tabular}

\section{Selecting pulsar candidates}

We are developing two different techniques to search for pulsars. Instead of scanning large parts of the sky for pulsating sources, we do a targeted search on 
candidate pulsars. These candidates are selected in two different ways using the WENSS.

\subsection{High polarization}

We select highly polarized objects from the WENSS. $60 \%$ of all known pulsars have a polarization degree larger than $20 \%$ at low frequencies. Because very few other objects have such a high polarization at these frequencies this is a good selection criterion. At this moment we are reducing the polarization data.

\subsection{Steep spectrum}

We determine spectral indices of WENSS-325 sources by comparing their fluxes with other surveys such as the WENSS-612 MHz survey and the VLA FIRST$1500 \mathrm{MHz}$ (Faint Images of the Radio Sky at Twenty-cm) survey. $60 \%$ of all known pulsars have spectral indices steeper than -1.5 , but there are also other objects with similarly steep spectra.

\section{Searching for pulsars}

Because we do not cover large parts of the sky we can observe each candidate for a relatively long time (typically half an hour). We know the integrated flux of the source so we adapt the settings of our filterbank in Westerbork such that we will be able to detect the pulsar, even if it has a short period and/or a high dispersion measure, and we adapt the reduction process to include an acceleration search in case the pulsar is in a close binary.

Recently the fast millisecond binary pulsar J0218+4232 has been found in a targeted search (Navarro et al. 1995). The radio source was already known for its steep spectrum and high polarization and therefore it was expected to be a pulsa.r. Because of its large dispersion measure $\left(61.2 \mathrm{pc} / \mathrm{cm}^{3}\right)$ and its short orbit (2 days) it was not detected in any untargeted survey before. Our systematic search will be more sensitive to these kinds of systems.

So far we have selected 72 candidates with spectra steeper than -1.5 from a WENSS-325 to WENSS-612 comparison. We have observed them but none showed pulsations. However most of these objects might be extended (and no pulsar) but unresolved in the low resolution WENSS maps

We expect better results by comparing WENSS-325 to FIRST-1500 and looking in the high resolution FIRST maps to see whether the source is extended or not. We have observed 16 of these point sources, but no pulsar has been found.

Acknowledgments. This research is supported by NWO under grant PGS 78-277.

\section{References}

Becker, R.H., White, L.W., Helfand, D.J., 1995, Ap.J, 450, 559

de Bruyn, A.G., 1996, in proc. IAU symp. 175, iñ press

Navarro, J., de Bruyn, A.G., Frail, D.A., Kulkarni, S.R., Lyne, A.G., 1995, ApJ, 455 , L55 